ARTICLE

\title{
Recent Advances in Computational Human Phantom for Monte Carlo Dose Calculation
}

\author{
Chan Hyeong KIM*, Jong Hwi JEONG and Yeon Soo YEOM \\ Department of Nuclear Engineering, Hanyang University \\ 17 Haengdang-dong, Seongdong-gu, Seoul 133-791, Korea
}

\begin{abstract}
In the present paper, an advanced type of a computational human phantom called PSRK-Man (Polygon Surface Reference Korean-Man) is introduced with its advantages over the traditional voxel phantom. This paper also discusses the computation speed issue of the surface phantom and the implementation of the direct accelerated geometry Monte Carlo (DAGMC) toolkit in Geant4, which makes the computation speed of the surface phantom by 20-30 times. With the computation speed problem solved, the surface phantom can be used in many applications, including medical imaging, accurate charged particle dosimetry, and radiation therapy simulations.
\end{abstract}

\section{KEYWORDS: polygon surface phantom, Monte Carlo, DAGMC, Geant4}

\section{Introduction}

The computational human phantom (CHP) is a numerical model of a human body that can be used in computer simulations. Figure 1 shows a brief history of CHPs. First, simple geometric forms such as spheres, cylinders, or boxes were used to represent the human body. Then, more complicated models, called stylized or mathematical phantom, were developed to represent the human body ${ }^{1)}$.

Then, finally, since 1984, people started to use the voxel phantoms ${ }^{1)}$. The voxel phantoms are developed using tomography images of human bodies, like CT or MRI, and therefore these describe the human bodies much more realistically than a stylized phantom.

The voxel phantoms, however, have some limitations. First, the voxel phantoms are not deformable and therefore it is very difficult, if not possible, to modify the posture of the phantom or the shape of the organs in the phantoms. The voxel phantoms also show stair-stepped surfaces and it is not suitable for medical imaging simulation because these steps will appear on simulated images which do not really exist on real images. It is also very difficult to model very thin or complicated structures such as oral mucosa, skin, eye balls, and internal ears.

On the other hand, a surface phantom, a polygon-mesh phantom or a NURBS phantom, does not have the aforementioned problems. Indeed, a surface phantom is freely deformable and therefore the shape of the phantom or internal organs can be easily changed. Four-dimensional (4D) Monte Carlo simulation is feasible; that is, we can transport the (radiation) particles during the movement of the organs in a patient due to cardiac or respiratory actions, or during the movement of a radiation worker in a radiation field. The surface phantom can easily model very thin structures such as oral mucosa and skin, which are

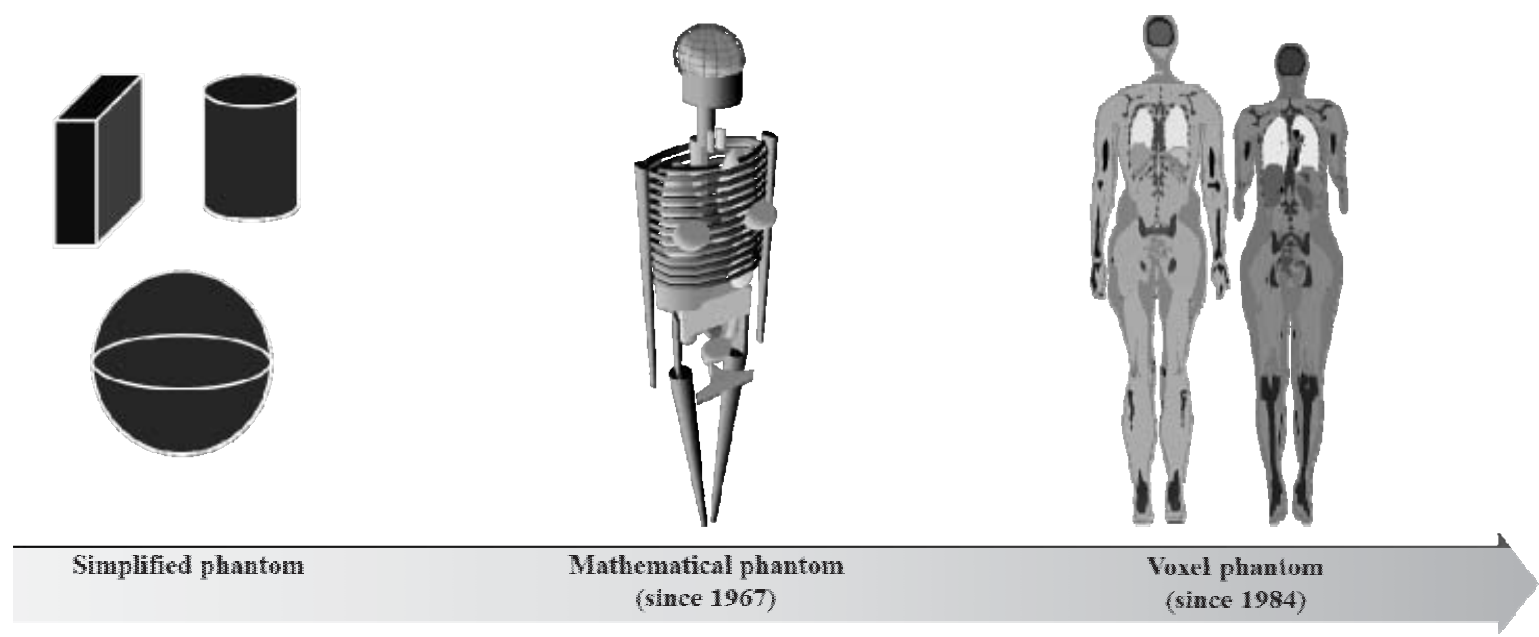

Fig. 1 Brief history of computational human phantoms.

*Corresponding Author, E-mail:chkim@hanyang.ac.kr

(C) 2012 Atomic Energy Society of Japan, All Rights Reserved. 

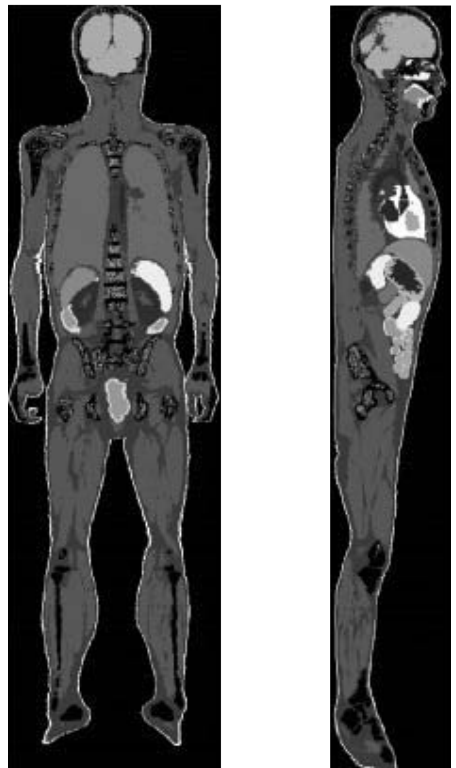

Fig. 2 VKH-Man voxel phantom.

radiosensitive and very important in radiation protection dosimetry. Complicated structures can be modeled to a great detail, including eye balls or internal ears, which are important organs in radiation therapies. The surface phantom shows smooth surface, which is good for medical imaging simulations like X-ray or CT simulations. The smooth surface is also beneficial for accurate dosimetry of charged particles including high-energy electrons and cosmic radiations.

These days, therefore, many researchers are trying to develop surface phantoms ${ }^{1-6)}$ and this paper will briefly describe a surface phantom called PSRK-Man (Polygon Surface Reference Korean-Man) that was developed in Korea to represent the adult male Korean workers ${ }^{7)}$ with the implementation of the direct accelerated geometry Monte Carlo (DAGMC) toolkit ${ }^{8)}$ that significantly improves the computation speed of the surface phantom.

\section{Surface phantom, PSRK-Man}

Recently, a surface phantom called PSRK-Man phantom was developed to represent adult Korean workers. To develop the surface phantom, first, a voxel phantom called VKH-Man ${ }^{9)}$ was developed using serially sectioned color slice images of a human body (Figure 2). Then, the VKH-Man voxel phantom was converted to a surface phantom. First, we performed surface rendering, using 3D-DOCTOR ${ }^{\mathrm{TM}}$ (Able Software Corp., Lexington, MA), to generate primitive polygon surfaces for the internal organs, bone structures, and body surface of the VKH-Man voxel phantom. Then, these primitive polygons were treated with refining procedures. For the most organs, except for the stomach, intestines, and blood vessels, the primitive surfaces were treated by the Smooth and Reduce Mesh processes in Rhinoceros ${ }^{\circledR} 4.0$ (McNeel, Seattle, WA). Then, the volumes of the organs were adjusted by the Offset Mesh process which was also used to define the walls of the organs. The refining process was mostly done with Rhinoceros ${ }^{\circledR} 4.0$,
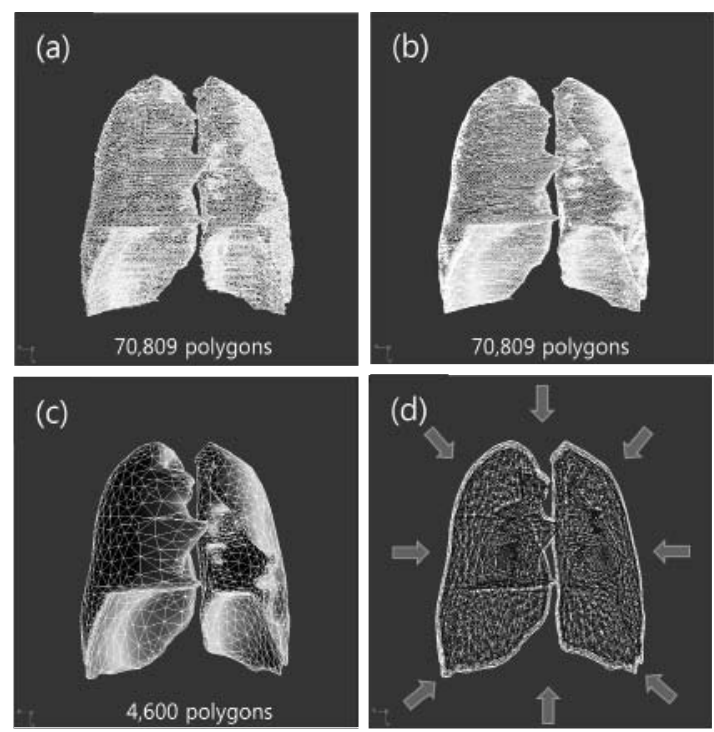

Fig. 3 Refining process of lung in Rhinoceros ${ }^{\circledR} 4$.0. (a) Raw polygon data from 3D-DOCTOR ${ }^{\mathrm{TM}}$, (b) Smooth, (c) Reduce Mesh, and (d) Offset Mesh.
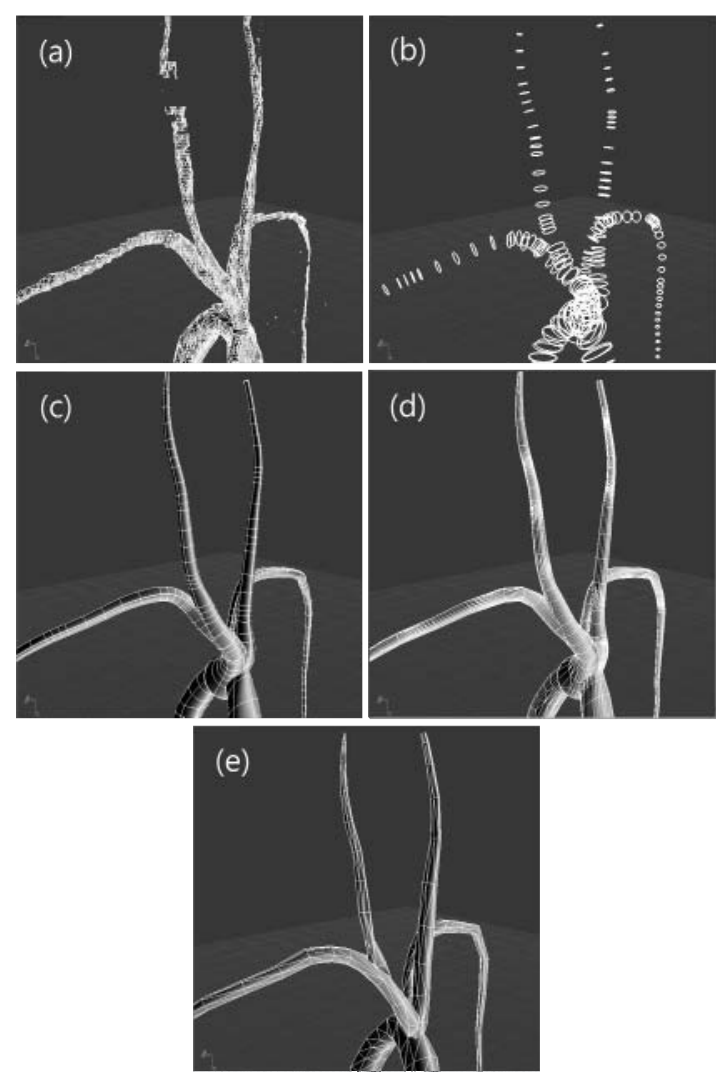

Fig. 4 Construction of blood vessel model. (a) raw polygon surfaces, (b) 3D contours, (c) NURBS surfaces, (d) polygon surfaces, and (e) reduce mesh.

except for some cleaning processes, for which Rapidform $^{\circledR} 2006$ (INUS, Seoul, Korea) was used. Figure 3 shows an example of the refining process.

For the stomach, intestines, and blood vessels, the primitive surfaces from the $3 \mathrm{D}-\mathrm{DOCTOR}{ }^{\mathrm{TM}}$ surface rendering was used 


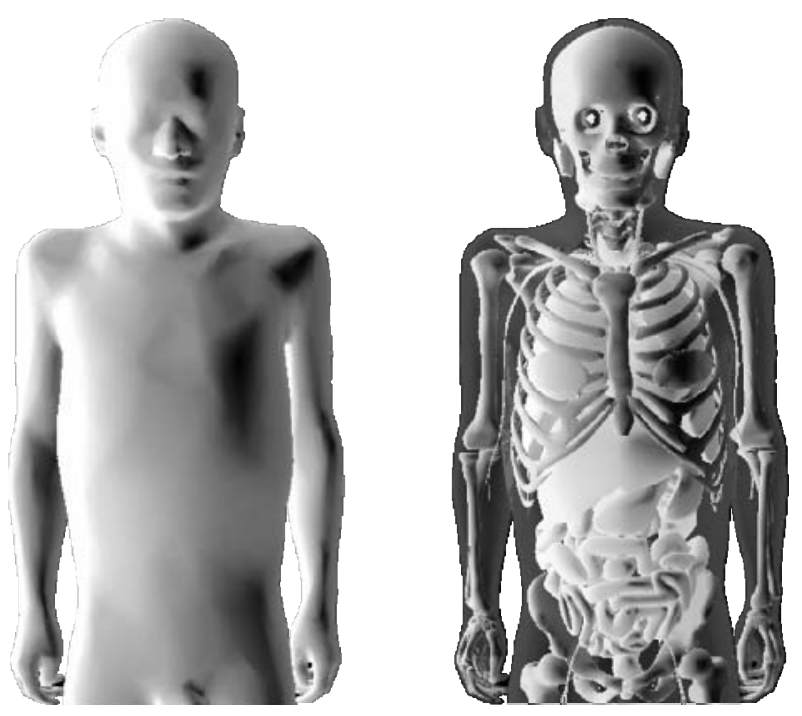

Fig. 5 PSRK-Man polygon surface phantom.

to generate $3 \mathrm{D}$ contours of these organs, and then the contours were used to generate NURBS surfaces. The NURBS surfaces were then converted to polygon surfaces. These polygon surfaces were finally treated with the Reduce Mesh and some cleaning processes. Figure 4 shows these processes for the blood vessel as an example.

Figure 5 shows the PSRK-Man phantom we developed as a surface phantom. The phantom is made of 120,850 polygons and the average size of the polygons is $0.51 \mathrm{~cm}^{2}$. The PSRK-Man phantom includes 27 radiosensitive organs and can be used for effective dose calculations following the ICRP 103 recommendation ${ }^{10)}$. The PSRK-Man phantom is indeed the first surface phantom that can be directly used in a Monte Carlo code without 'voxelization' process ${ }^{7}$.

The PSRK-Man phantom was ported to the Geant4 Monte Carlo code by using the G4TessellatedSolid class and the phantom was used for Monte Carlo dose calculations. First, the surface phantom PSRK-Man was used to calculate dose conversion coefficients (DCCs) for photons and the calculated values were compared with those of the HDRK-Man ${ }^{11)}$ voxel phantom. The result showed that the calculated values of these two phantoms were very close to each other, which means the surface phantom produces the right values. The comparison of the computation times of these two phantoms, however, revealed a significant problem for the surface phantom. Table 1 compares the computation speed and shows that the PSRK-Man phantom is about 70-150 times slower than the HDRK-Man phantom for photons.

\section{Computation speed and DagSolid}

To solve the computation speed problem of the surface phantom, we have implemented the DAGMC toolkit and developed a new solid class in Geant4. The new solid class was derived from the G4TessellatedSolid class which was used in a previous study for the PSRK-Man phantom. Some mandatory member functions (i.e., the function to calculate distance from particle position to boundaries, the function to decide if the particle position is inside or outside of the solid volume, and the function to calculate outwards
Table 1 Comparison of computation time for the same number of the primary incident photon $\left(=10^{7}\right)$ for AP geometry.

\begin{tabular}{lcccr}
\hline & \multicolumn{2}{c}{ Computation time (hh:mm:ss) } & & factor \\
\cline { 2 - 3 } \cline { 5 - 5 }$(\mathrm{MeV})$ & $\begin{array}{c}\text { HDRK-Man } \\
\text { voxel (a) }\end{array}$ & $\begin{array}{c}\text { PSRK-Man } \\
\text { polygon (b) }\end{array}$ & & \\
& $0: 27: 10$ & $65: 17: 33$ & & 144.2 \\
\hline 0.015 & $0: 36: 01$ & $73: 54: 04$ & & 123.1 \\
0.03 & $0: 45: 54$ & $86: 10: 14$ & & 112.6 \\
0.05 & $1: 16: 14$ & $95: 42: 08$ & & 75.3 \\
0.08 & $0: 58: 07$ & $101: 16: 00$ & & 104.5 \\
0.2 & $0: 58: 16$ & $99: 40: 11$ & & 102.6 \\
0.4 & $0: 50: 44$ & $98: 56: 25$ & & 117.0 \\
0.8 & $0: 54: 37$ & $96: 12: 04$ & & 105.7 \\
2 & $0: 53: 58$ & $99: 16: 57$ & & 110.4 \\
8 & $0: 56: 20$ & $100: 13: 50$ & \\
10 & & & 106.8 \\
\hline
\end{tabular}

pointing unit normal vector) of the derived solid class were

Table 2 Comparison of computation times between G4TessellatedSolid class and DagSolid class for PSRK-Man in the same condition with Table 1.

\begin{tabular}{|c|c|c|c|}
\hline \multirow[b]{2}{*}{$(\mathrm{MeV})$} & \multicolumn{2}{|c|}{ Computation time (hh:mm:ss) } & \multirow{2}{*}{$\begin{array}{l}\text { factor } \\
(\mathrm{a} / \mathrm{b})\end{array}$} \\
\hline & $\begin{array}{l}\text { G4Tessellated- } \\
\text { Solid } \\
\text { (a) }\end{array}$ & $\begin{array}{l}\text { DagSolid } \\
\text { (b) }\end{array}$ & \\
\hline 0.015 & $65: 17: 33$ & $2: 15: 07$ & 29.0 \\
\hline 0.03 & $73: 54: 04$ & $2: 33: 53$ & 28.8 \\
\hline 0.05 & $86: 10: 14$ & $2: 58: 04$ & 29.0 \\
\hline 0.08 & $95: 42: 08$ & $3: 15: 18$ & 29.4 \\
\hline 0.2 & $101: 16: 00$ & $3: 30: 44$ & 28.8 \\
\hline 0.4 & $99: 40: 11$ & $3: 38: 33$ & 27.4 \\
\hline 0.8 & $98: 56: 25$ & $3: 43: 02$ & 26.6 \\
\hline 2 & $96: 12: 04$ & $3: 58: 18$ & 24.2 \\
\hline 8 & $99: 16: 57$ & $4: 49: 28$ & 20.6 \\
\hline 10 & $100: 13: 50$ & $4: 47: 04$ & 20.9 \\
\hline
\end{tabular}

then replaced with the functions in the linked library of the DAGMC toolkit. The key algorithm of DAGMC toolkit is based on the oriented bounding box (OBB) tree, the use of which avoids calculating the distances from a particle position to all of the facets of the polygon-surface geometries and thus significantly save the computation time. The new Geant 4 solid class developed in the present study was named DagSolid and the detailed discussion on the development of the solid will be given in a dedicated paper. Table 2 shows that the PSRK-Man using the DagSolid class is about 20-30 times faster than the case using the original G4TessellatedSolid class, which is a significant improvement. However, there is still about 5 
times of difference between the computation speed of the surface and voxel phantoms, which we believe can be overcome by developing a dedicated navigator in Geant4 for the surface phantom. The computation speed of the surface phantom, which uses polygon surfaces to define the organs and skins, can be also effectively improved by using the general-purpose computing on graphics processing units (GPGPU) programming.

\section{Conclusions}

This paper briefly introduces an advanced type of computational human phantom, the PSRK-Man surface phantom, and the DAGMC acceleration toolkit, which is used to improve the computation speed of the surface phantom based on the oriented bounding box (OBB) tree. If the computation speed problem is addressed successfully, then there will be a plenty of applications for the surface phantom. Specifically, 4D Monte Carlo simulation for accurate dose calculation and imaging seems a very promising research area for a surface phantom. The topic is very challenging requiring expertise on both computational human phantom and 4D Monte Carlo simulation technologies, but still it is a very important and very promising research area and if we are successful, we can transport particles while accurately modeling the respiratory and cardiac motion of the patient, which will significantly improve the accuracy of radiation therapy and imaging simulations.

\section{Acknowledgment}

This research was supported by National Nuclear R\&D Program through the National Research Foundation of Korea (NRF) funded by the Ministry of Education, Science and Technology (2011-0002090, 2010-0023825)

\section{References}

1) W. P. Segars and B. M. Tsui, "Study of the efficacy of respiratory gating in myocardial SPECT using the new 4D NCAT phantom," IEEE Trans. Nucl. Sci. 49 675-679 (2002).

2) C. Lee, D. Lodwick, D. Hasenauer, J. L. Williams, C. Lee and W. E. Bolch, "Hybrid computational phantoms of the male and female newborn patient - NURBS-based whole-body models," Phys. Med. Biol., 52, 3309-3333 (2007).

3) $\mathrm{X} . \mathrm{G} . \mathrm{Xu}, \mathrm{V}$. Taranenko, J. Zhang and C. Shi, "A boundary-representation method for designing whole-body radiation dosimetry models pregnant females at the ends of three gestational periods-RPI-P3, -P6 and -P9," Phys. Med. Biol., 52 7023-7044 (2007).

4) J. Zhang, Y. H. Na, P. F. Caracappa and X. G. Xu, "RPI-AM and RPI-AF, a pair of mesh-based, size-adjustable adult male and female computational phantoms using ICRP-89 parameters and their calculations for organ doses from monoenergetic photon beams," Phys. Med. Biol., 54, 5885-5908 (2009).

5) V. F. Cassola, V. J. de Melo Lima, R. Kramer and H. J. Khoury, "FASH and MASH: female and male adult human phantoms based on polygon mesh surfaces: I. Development of the anatomy," Phys. Med. Biol., 55, 133-162 (2010).

6) W. P. Segars, G. Sturgeon, S. Mendonca, Jason Grimes and B. M. W. Tsui, "4D XCAT phantom for multimodality imaging research," Med Phys., 37(9), 4902-4915 (2010).

7) C. H. Kim, J. H. Jeong, W. E. Bolch, K.-W. Cho and S. B. Hwang, "Polygon-surface reference Korean male phantom (PSRK-Man) and its direct implementation in Geant4 Monte Carlo simulation," Phys. Med. Biol., 56, 3137-3161 (2011).

8) T. J. Tautges, P. P. H. Wilson, J. A. Kraftcheck, B. M. Smith and D. L. Henderson, "Acceleration techniques for direct use of CAD-Based geometries in Monte Carlo radiation transport," Proceedings of the 2009 International Conference on Mathematics, Computational Methods \& Reactor Physics (M\&C 2009), May 3-7, 2009, Saratoga Springs, NY, USA

9) S. H. Choi, J. H. Jeong, S. Cho, M. S. Chung, H. D. Huh, W. C. Kim K.-W. Cho and C. H. Kim, "Construction of a high-quality voxel model VKH-Man using serially sectioned images from Visible Korean Human project in Korea," J. Nucl. Sci. Technol. S5, 179-182 (2008).

10) International Commission on Radiological Protection, The 2007 recommendations of the International Commission on Radiological Protection, ICRP Publication 103, Elsevier Publishing, New York (2008).

11) C. H. Kim, S. H. Choi, J. H. Jeong, C. Lee and M. S. Chung, "HDRK-Man: a whole-body voxel model based on high-resolution color slice images of a Korean adult male cadaver," Phys. Med. Biol., 53, 4093-4106 (2008). 